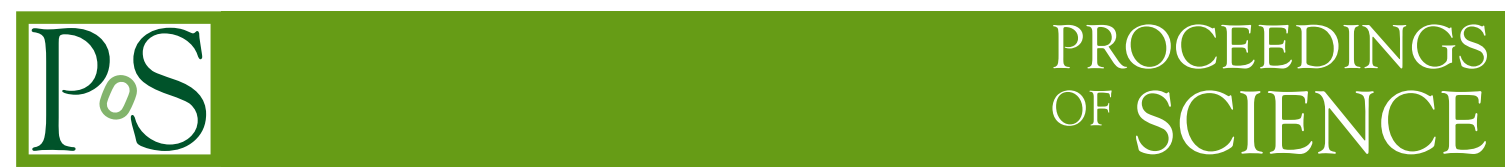

\title{
Recent developments in the physics of light quarks
}

\author{
Heinrich Leutwyler* \\ University of Bern \\ E-mail: Leutwylerditp.unibe.ch
}

My talk was dedicated to the memory of Jan Stern. The brief account given below focuses on the progress achieved in the determination of the $\pi \pi \mathrm{S}$-wave scattering lengths, both experimentally and with light dynamical quarks on a lattice. In view of the excellent agreement, we can conclude that (a) the expansion in powers of the two lightest quark masses represents a very efficient tool for the analysis of the low energy structure of QCD and (b) the size of the energy gap of QCD is governed by the order parameter of lowest dimension, the quark condensate.

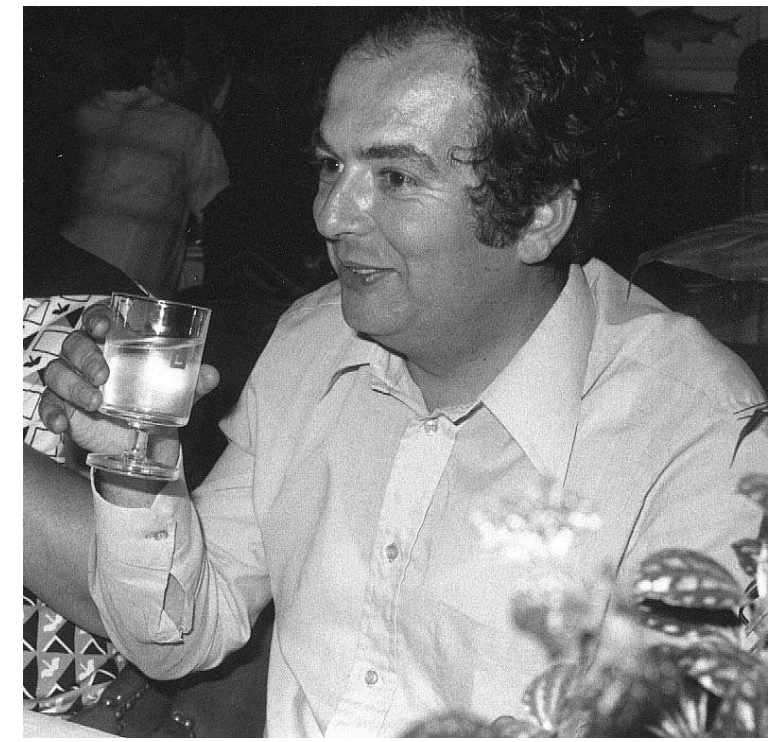

In memoriam Jan Stern, 29. 6. 1942 - 2. 7. 2008

8th Conference Quark Confinement and the Hadron Spectrum September 1-6, 2008

Mainz. Germany

\footnotetext{
${ }^{*}$ Speaker.
} 


\section{Introduction}

In view of the limited space available, the present report only summarizes the first part of my talk, which dealt with the remarkable progress achieved with light dynamical quarks on the lattice and with the low energy precision experiments concerning the $\pi \pi \mathrm{S}$-wave scattering lengths. A more detailed account, which also outlines recent developments in the dispersive analysis of the low energy structure of QCD, is given in [1].

At low energies, the main characteristic of QCD is that the energy gap is remarkably small, $M_{\pi} \simeq 140 \mathrm{MeV}$. More than 10 years before the discovery of QCD, Nambu [2] found out why that is so: the gap is small because the strong interaction has an approximate chiral symmetry. Indeed, QCD does have this property: for yet unknown reasons, two of the quarks happen to be very light. The symmetry is not perfect, but nearly so: $m_{u}$ and $m_{d}$ are tiny. The mass gap is small because the symmetry is "hidden" or "spontaneously broken": for dynamical reasons, the ground state of the theory is not invariant under chiral rotations, not even approximately. The spontaneous breakdown of an exact Lie group symmetry gives rise to strictly massless particles, "Goldstone bosons". In QCD, the pions play this role: they would be strictly massless if $m_{u}$ and $m_{d}$ were zero, because the symmetry would then be exact. The only term in the Lagrangian of QCD that is not invariant under the group $\mathrm{SU}(2) \times \mathrm{SU}(2)$ of chiral rotations is the mass term of the two lightest quarks, $m_{u} \bar{u} u+m_{d} \bar{d} d$. This term equips the pions with a mass. Although the theoretical understanding of the ground state is still poor, we do have very strong indirect evidence that Nambu's conjecture is right - we know why the energy gap of QCD is small.

\section{Lattice results for $M_{\pi}$ and $F_{\pi}$}

As pointed out by Gell-Mann, Oakes and Renner [3], the square of the pion mass is proportional to the strength of the symmetry breaking, $M_{\pi}^{2} \propto\left(m_{u}+m_{d}\right)$. This property can now be checked on the lattice, where - in principle - the quark masses can be varied at will. In view of the fact that in these calculations, the quarks are treated dynamically, the quality of the data is impressive. The masses are sufficiently light for $\chi \mathrm{PT}$ to allow a meaningful extrapolation to the quark mass values of physical interest. The results indicate that the ratio $M_{\pi}^{2} /\left(m_{u}+m_{d}\right)$ is nearly constant out to values of $m_{u}, m_{d}$ that are about an order of magnitude larger than in nature. According to Gell-Mann, Oakes and Renner, this ratio is related to the quark condensate. The Banks-Casher relation, which connects the quark condensate with the spectral density of the Dirac operator at small eigenvalues [4], is now also accessible to a numerical evaluation on the lattice [5].

The Gell-Mann-Oakes-Renner relation corresponds to the leading term in the expansion of $M_{\pi}^{2}$ in powers of $m_{u}$ and $m_{d}$ (mass of the strange quark kept fixed at the physical value). At next-toleading order, the expansion contains a logarithm:

$$
M_{\pi}^{2}=M^{2}\left\{1+\frac{M^{2}}{32 \pi^{2} F_{\pi}^{2}} \ln \frac{M^{2}}{\Lambda_{3}^{2}}+O\left(M^{4}\right)\right\}
$$

where $M^{2} \equiv B\left(m_{u}+m_{d}\right)$ stands for the term linear in the quark masses. Chiral symmetry fixes the coefficient of the logarithm in terms of the pion decay constant $F_{\pi} \simeq 92.2 \mathrm{MeV}$, but does not determine the scale $\Lambda_{3}$. An estimate for this scale was obtained more than 20 years ago [6], 
on the basis of the SU(3) mass formulae for the pseudoscalar octet: $\bar{\ell}_{3} \equiv \ln \Lambda_{3}^{2} / M_{\pi}^{2}=2.9 \pm 2.4$. Several collaborations have now managed to determine the scale $\Lambda_{3}$ on the lattice - for an overview, I refer to [7]. The result of the RBC /UKQCD collaboration, $\bar{\ell}_{3}=3.13 \pm 0.33_{\text {stat }} \pm 0.24_{\text {syst }}$, for instance, which concerns $2+1$ flavours and includes an estimate of the systematic errors, is perfectly consistent with the number quoted above, but considerably more accurate.

The expansion of $F_{\pi}$ in powers of $m_{u}, m_{d}$ also contains a logarithm at NLO. The coupling constant relevant in that case is denoted by $\bar{\ell}_{4}$. A couple of years ago, we obtained a rather accurate result for this quantity, from a dispersive analysis of the scalar form factor: $\bar{\ell}_{4}=4.4 \pm 0.4$ [8] (for details, I refer to [9]). The lattice determinations of $\bar{\ell}_{4}$ have reached comparable accuracy and are consistent with the dispersive result [7].

Concerning the expansion in powers of $m_{s}$, however, the current situation leaves much to be desired. While some of the lattice results indicate, for instance, that the violations of the OkuboZweig-Iizuka rule in the quark condensate and in the decay constants are rather modest, others point in the opposite direction. In view of the remarkable progress being made with the numerical simulation of light quarks, I am confident that the dust will settle soon, so that the effective coupling constants that govern the dependence of the various quantities of physical interest on $m_{s}$ can reliably be determined, to next-to-next-to-leading order of the chiral expansion.

\section{Consequences for the $\pi \pi$ scattering lengths}

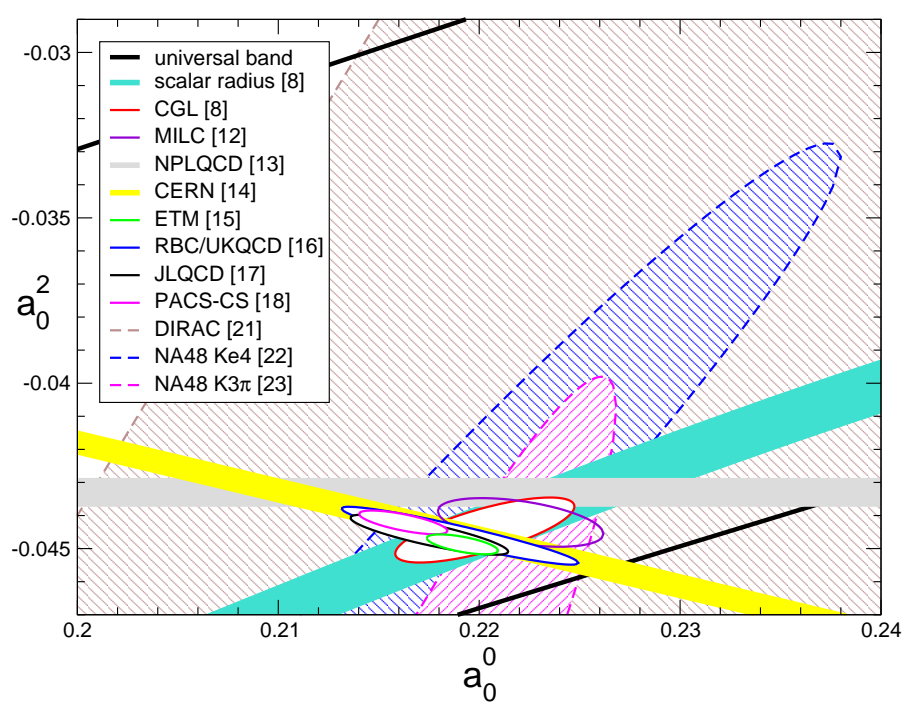

Figure 1: Lattice results for $\bar{\ell}_{3}, \bar{\ell}_{4}$ [12-18], converted into S-wave $\pi \pi$ scattering lengths, compared with $K_{e 4}$ and $K_{3 \pi}$ data and with the prediction in [8], which relied on $\mathrm{SU}(3)$ for $\bar{\ell}_{3}$ and on a dispersive evaluation of the scalar radius for $\bar{\ell}_{4}$. Figure prepared in the framework of Flavianet, in collaboration with G. Colangelo.

The hidden symmetry not only controls the size of the energy gap, but also determines the interaction of the Goldstone bosons at low energies, among themselves, as well as with other hadrons. In particular, as pointed out by Weinberg [10], the leading term in the chiral expansion of the Swave $\pi \pi$ scattering lengths (tree level of the effective theory) is determined by the pion decay con- 
stant. In the meantime, the chiral perturbation series has been worked out to NNLO and, matching the chiral and dispersive representations of the scattering amplitude, a sharp prediction for the scattering lengths was obtained a couple of years ago: $a_{0}^{0}=0.220(5), a_{0}^{2}=-0.0444(10)$ [8]. The error bars are dominated by the uncertainties in the estimates used for the effective coupling constants $\bar{\ell}_{3}, \bar{\ell}_{4}$, which were quoted above. Since recent work on the lattice has reduced these uncertainties - particularly in the case of $\bar{\ell}_{3}$ - the predictions for the scattering lengths have now become even sharper. This is illustrated in Fig. 1, where the various lattice results for $\bar{\ell}_{3}, \bar{\ell}_{4}$ are converted into results for $a_{0}^{0}, a_{0}^{2}$, using the formulae in [11]. The ellipses indicate the corresponding $1 \sigma$ contours, obtained by summing up all errors in square, including an estimate for the neglected NNLO corrections in the relation between the scattering lengths and $\bar{\ell}_{3}, \bar{\ell}_{4}$ (some of the lattice results shown concern QCD with $N_{f}=2$ and are thus subject to an unknown systematic error). Indeed, there are tensions among the lattice results, but the plot shows that all of these are within one standard deviation of the prediction obtained on the basis of $\chi \mathrm{PT}$ (red ellipse, taken from [8]). Note the scale: the width of the figure corresponds to deviations from the central prediction for $a_{0}^{0}$ of less than $10 \%$. NPLQCD, for instance, quotes the outcome for the exotic scattering length $a_{0}^{2}$ to an accuracy of $1 \%$, systematic errors included [13]. The result is obtained by analyzing mixed-action data by means of $\chi$ PT to NLO.

\section{Low energy precision experiments}

I add a few remarks concerning the experimental information about the scattering lengths.

1. Production experiments such as $\pi N \rightarrow \pi \pi N, J / \psi \rightarrow \pi \pi \omega, \ldots$ provide valuable information about the $\pi \pi$ phase shifts in the intermediate energy region, but since the pions are not produced in vacuo, the analysis is complicated - the uncertainties in the results for the scattering lengths are much too large for these experiments to be of interest in the present context.

2. In principle, the decay $K \rightarrow \pi \pi$ can be used to measure the phase difference $\delta_{0}^{0}-\delta_{0}^{2}$ at the kaon mass. Unfortunately, however, the $\Delta I=\frac{1}{2}$ rule implies that the result for the phase difference is subject to unusually large isospin breaking effects. In the past, work on this problem invariably led to a value for the phase difference that is too large, presumably because isospin breaking was not properly accounted for. Only rather recently, Cirigliano, Ecker, Neufeld and Pich have performed a complete analysis of these transitions, based on $\chi$ PT to NLO [19]. Unfortunately, however, the discrepancy persists. I conclude that, at the present level of our understanding, the uncertainties associated with isospin breaking are too large for the decay $K \rightarrow \pi \pi$ to provide useful information about the low energy structure of QCD.

3. The low energy theorem for the scalar radius of the pion correlates the two S-wave scattering lengths to a narrow strip [8]. If this correlation is used, together with the corrections for isospin breaking obtained in [22], the $K_{e 4}$ data determine $a_{0}^{0}$ to the same precision as the theoretical prediction and hit the nail on the head: $a_{0}^{0}=0.220(5)(2)$ [23].

4. As pointed out by Cabibbo [24], the cusps occurring near threshold in decays of kaons into three pions can be used to measure the combination $a_{0}^{0}-a_{0}^{2}$ of scattering lengths. A preliminary analysis of the $2003+2004$ data collected on $K_{3 \pi}$ decay at NA48/2 is reported in [25]. Using the framework derived in [26], and the low energy theorem for the scalar radius, these 
data imply $a_{0}^{0}-a_{0}^{2}=0.266 \pm 0.003_{\text {stat }} \pm 0.002_{\text {syst }} \pm 0.001_{\text {ext }}$, thus subjecting the $\chi$ PT prediction, $a_{0}^{0}-a_{0}^{2}=0.265 \pm 0.004[8]$, to a very stringent test.

I conclude that the low energy precision measurements as well as the results obtained on the lattice consolidate the picture developed on the basis of $\chi \mathrm{PT}$ : the expansion of the square of the pion mass in powers of $m_{u}, m_{d}$ is dominated by the leading term, which is proportional to the quark condensate. The NLO contributions are now known rather accurately - as expected, they are tiny.

\section{References}

[1] H. Leutwyler, in Proc. QCD 08, Montpellier, France, July 2008, ed. S. Narison, arXiv:0809.5053.

[2] Y. Nambu, Phys. Rev. Lett. 4 (1960) 380.

[3] M. Gell-Mann, R. J. Oakes and B. Renner, Phys. Rev. 175 (1968) 2195.

[4] T. Banks and A. Casher, Nucl. Phys. B 169 (1980) 103.

[5] L. Giusti, talk given at this conference.

[6] J. Gasser and H. Leutwyler, Phys. Lett. B 125 (1983) 325; Annals Phys. 158 (1984) 142.

[7] G. Ecker and S. Necco, talks given at this conference.

[8] G. Colangelo, J. Gasser and H. Leutwyler, Nucl. Phys. B 603 (2001) 125.

[9] B. Ananthanarayan et al., Phys. Lett. B 602 (2004) 218.

[10] S. Weinberg, Phys. Rev. Lett. 17 (1966) 616.

[11] H. Leutwyler, in Proc. Chiral Dynamics, Theory \& Experiment, Durham/Chapel Hill, NC, USA, eds. M. W. Ahmed et al., World Scientific, Singapore (2007), p.17, hep-ph/0612112.

[12] C. Bernard et al. [MILC Collaboration], PoS LAT2007 090.

[13] S. R. Beane et al. [NPLQCD Collaboration], Phys. Rev. D 77 (2008) 014505;

A. Walker-Loud, talk given at this conference.

[14] L. Del Debbio, L. Giusti, M. Lüscher, R. Petronzio and N. Tantalo, JHEP 0702 (2007) 056.

[15] Ph. Boucaud et al. [ETM Collaboration], Phys. Lett. B 650 (2007) 304.

[16] C. Allton et al. [RBC-UKQCD Collaboration], arXiv:0804.0473.

[17] J. Noaki et al. [JLQCD and TWQCD Collaborations], Phys. Rev. Lett. 101 (2008) 202004.

[18] D. Kadoh et al. [PACS-CS Collaboration], PoS LAT2007 109.

[19] V. Cirigliano, G. Ecker, H. Neufeld and A. Pich, Phys. Rev. Lett. 91 (2003) 162001; Eur. Phys. J. C 33 (2004) 369.

[20] V. Cirigliano, C. Gatti, M. Moulson, M. Palutan, for the FlaviaNet Kaon Working Group, arXiv:0807.5128.

[21] B. Adeva et al. [DIRAC Collaboration], Phys. Lett. B 619 (2005) 50.

[22] G. Colangelo, J. Gasser and A. Rusetsky, arXiv:0811.0775;

[23] B. Bloch-Devaux, talk given at this conference.

[24] N. Cabibbo, Phys. Rev. Lett. 93 (2004) 121801; N. Cabibbo and G. Isidori, JHEP 0503 (2005) 021.

[25] D. Madigozhin, talk given at Flavianet Kaon Workshop, Anacapri 2008.

[26] G. Colangelo, J. Gasser, B. Kubis and A. Rusetsky, Phys. Lett. B 638 (2006) 187. 